\title{
Recurrence Relations for Knot Polynomials of Twist Knots
}

\author{
Kemal Taşköprï ${ }^{1 *}$ and Zekiye Şevval Sinan ${ }^{1}$ \\ ${ }^{1}$ Department of Mathematics, Bilecik Şeyh Edebali University, Bilecik, Turkey \\ * Corresponding author
}

\section{Article Info}

Keywords: Knot polynomial, Oriented knot, Unoriented knot, Twist knot

2010 AMS: 57M25, 57M27

Received: 08 January 2021

Accepted: 17 March 2021

Available online: 20 March 2021

\begin{abstract}
This paper gives HOMFLY polynomials and Kauffman polynomials $L$ and $F$ of twist knots as recurrence relations, respectively, and also provides some recursive properties of them.
\end{abstract}

\section{Introduction}

The knot polynomials are the most practical knot invariants for distinguishing knots from each other, where the coefficients of polynomials represent some properties of the knot. The first of the polynomial invariants is the Alexander polynomial [1] with one variable for oriented knots and links. There are generalizations of the Alexander polynomial and its Conway version [2], see [3]- [5]. Another important knot polynomial with one variable for oriented knots and links is the Jones polynomial [6]. Both the Jones polynomial was defined with new methods [7,8] and studies were conducted on generalizations of the Jones polynomial [9]- [11]. One of the most important generalized polynomials is the HOMFLY polynomial [11]- [13] with two variable. The Alexander and Jones polynomials are special cases of the HOMFLY polynomial. For unoriented knots and links, there are the polynomials such as the BLM/Ho polynomial $[14,15]$ with one variable and the Kauffman polynomial $F$ [16] with two variable whose primary version Kauffman polynomial $L$ is an invariant of regular isotopy for unoriented knots and links. Both the Jones and the BLM/Ho polynomials are special cases of the Kauffman polynomial $F$.

The HOMFLY polynomial or HOMFLY-PT polynomial whose name is an acronym for its discoverers' last names is inspired by the Jones polynomial. The HOMFLY polynomial $P_{K}(a, z)$ is two variables Laurent polynomial for the oriented link diagram $K . P_{K}(a, z)$ is an ambient isotopy invariant of the link $K$ determined by the following axioms:

$$
\begin{aligned}
a^{-1} P_{K_{+}}(a, z)-a P_{K_{-}}(a, z) & =z P_{K_{0}}(a, z) \\
P_{\bigcirc}(a, z) & =1,
\end{aligned}
$$

where $K_{+}, K_{-}$and $K_{0}$ are skein diagrams drawn in Figure 1.1 and $\bigcirc$ is any diagram of the unknot.

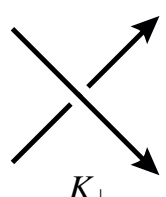

$K_{+}$

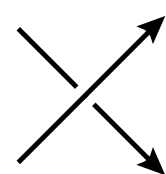

K

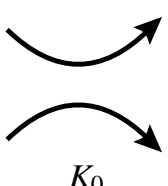

$K_{0}$

Figure 1.1: Skein Diagrams 
From the axioms (1.1) and (1.2), it is obtained that $a^{-1}-a=z P_{\bigcirc}$ or $\delta=\left(a^{-1}-a\right) z^{-1}$ with $\delta=P_{\bigcirc}$, where $\bigcirc \bigcirc$ is trivial link with two components. If $\bigcirc \mu$ is a trivial $\mu$-component link, then $P_{\bigcirc_{\mu}}(a, z)=\delta^{\mu-1}$. Also, $P_{K^{*}}(a, z)=P_{K}\left(a^{-1},-z\right)$, where $K^{*}$ is the mirror image of $K$.

In 1987, L. Kauffman [16, 17] discovered a new polynomial, denoted by $L$, which specializes to the bracket polynomial [7]. The Kauffman polynomial $L(a, x)$ is a two-variable Laurent polynomial for the unoriented link diagram $K . L(a, x)$ is a regular isotopy invariant of the link $K$ satisfying the following axioms:

$$
\begin{aligned}
L_{K^{+}}(a, x)+L_{K^{-}}(a, x) & =x\left(L_{K^{0}}(a, x)+L_{K^{\infty}}(a, x)\right), \\
L_{\bigcirc}(a, x) & =1 \\
L_{D^{+}}(a, x) & =a L_{D^{0}}(a, x) \\
L_{D^{-}}(a, x) & =a^{-1} L_{D^{0}}(a, x)
\end{aligned}
$$

where $K^{+}, K^{-}, K^{0}$ and $K^{\infty}$ are unoriented diagrams drawn in Figure 1.2, $\bigcirc$ is any diagram of unknot and $D^{+}, D^{-}$and $D^{0}$ are unoriented diagrams drawn in Figure 1.3.
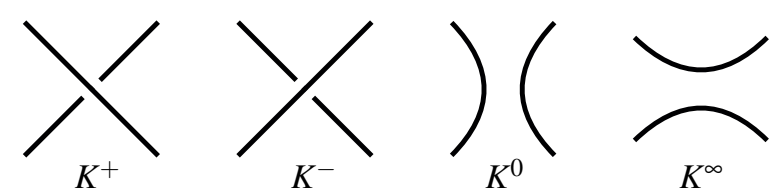

Figure 1.2: Crossings and splits
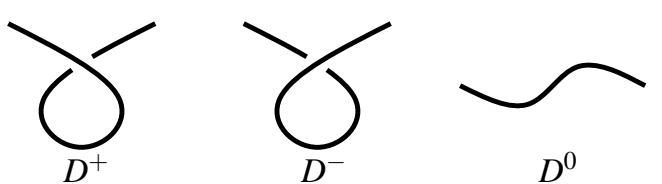

Figure 1.3: Diagrams related to Reidmeister moves of type I

The Kauffman polynomial $F$ for oriented link diagram $K$ by the formula [17]

$$
F_{K}(a, x)=a^{-w(K)} L_{K}(a, x)
$$

where $L_{K}$ is defined on oriented link diagrams by forgetting the orientation and $w(K)$ denotes the writhe of oriented link diagram $K(w(K)$ is the sum of all crossing signs of $K)$. Then the polynomial $F_{K}(a, x)$ is a Laurent polynomial invariant of ambient isotopy. From the axioms (1.3) and (1.5), it is obtained that $L_{\bigcirc}(a, x)=\left(a+a^{-1}\right) x^{-1}-1$ or $\delta=\left(a+a^{-1}\right) x^{-1}-1$ with $\delta=L_{\bigcirc}(a, x)$, where $\bigcirc \bigcirc$ is trivial link with two components. If $\bigcirc_{\mu}$ is a trivial $\mu$-component link, then $L_{\bigcirc \mu}(a, x)=$ $F_{\bigcirc_{\mu}}(a, x)=\delta^{\mu-1}$. Also, $L_{K^{*}}(a, x)=L_{K}\left(a^{-1}, x\right)$ and $F_{K^{*}}(a, x)=F_{K}\left(a^{-1}, x\right)$, where $K^{*}$ is the mirror image of link $K$.

The twist knots, which obtained by twisting a closed-loop repeatedly and then linking the ends together, are an essential class of knots. It could be found out lots of studies about their knot invariants (See [18]- [25] and others). Here, a twist knot is regarded with a clasp and right-handed $n$-half twists as drawn in Figure 2.1. Besides, the knot polynomials of some classes of knots and links were studied to give recursive formulas [26]- [31].

In this paper, it is aimed that deriving the recurrence relations for the HOMFLY polynomials of the oriented twist knots and the Kauffman polynomials $L$ and $F$ of the unoriented twist knots. While the HOMFLY polynomial and the Kauffman polynomials $L$ and $F$ of twist knots are defined as fourth-order recurrence relations, the $(2, n)$-torus link diagrams are encountered and their mentioned knot polynomials are utilized for some results. Also, some recursive properties of these relations are examined and it is provided the generating functions, the general solutions and the explicit forms.

\section{Oriented and unoriented knot polynomials of twist knots}

\subsection{HOMFLY polynomials of twist knots}

Suppose that $\mathscr{K}_{n}$ is an oriented digram of twist knot drawn in Figure 2.1, $\mathscr{K}_{(2, n)}$ is an oriented digram of $(2, n)$-torus link drawn in Figure 2.2 and $P_{n}$ denotes the HOMFLY polynomial of $\mathscr{K}_{n}$ instead of $P_{\mathscr{K}_{n}}(a, z)$ for simplicity. 


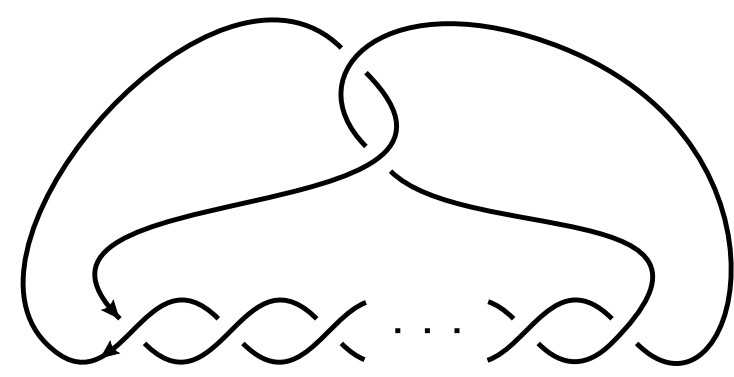

Figure 2.1: The twist knot with a clasp and right-handed $n$-half twists

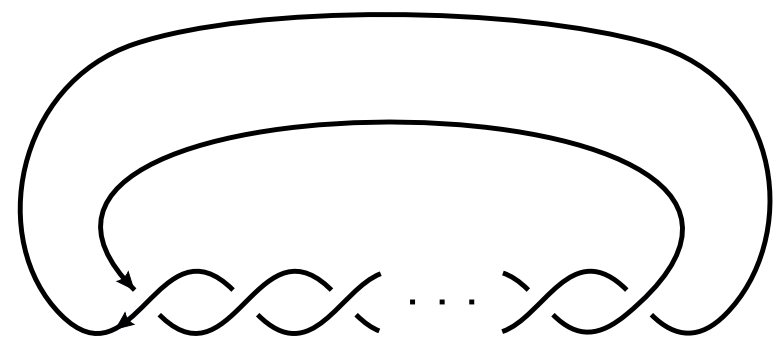

Figure 2.2: $(2, n)$-torus link

Theorem 2.1. The HOMFLY polynomial of twist knot $\mathscr{K}_{n}$ satisfies the following relations:

$$
P_{n}=\left(a^{2}+1\right) P_{n-2}-a^{2} P_{n-4}, \quad n \geq 4
$$

and

$$
P_{n}= \begin{cases}a z P_{\mathscr{K}_{(2, n+1)}}+a^{2} & \text { if } n \text { is odd }, \\ -a^{-1} z P_{\mathscr{K}_{(2, n)}}+a^{-2} & \text { if } n \text { is even } .\end{cases}
$$

Proof. Let the skein operations be applied to a designated half twist of the oriented diagram $\mathscr{K}_{n}$. If the crossing is switched, the resulting diagram is $\mathscr{K}_{n-2}$ twist knot by the second Reidemeister move. Then, if the crossing is smoothed, the resulting diagram is (2,2)-torus link, i.e. Hopf link, obtained by applying the first Reidemeister move $n-1$ times. Notice that, if $n$ is odd, all crossings of the (2,2)-torus link are right-handed with counter-directed strands and if $n$ is even, all crossings of the $(2,2)$-torus link are left-handed with same-directed strands. Hence, from the axiom (1.1), the following equations are obtained as

$$
P_{n}=a z P_{\mathscr{K}_{(2,2)}}+a^{2} P_{n-2}
$$

and

$$
P_{n-2}=a z P_{\mathscr{K}_{(2,2)}}+a^{2} P_{n-4}
$$

Thus, the recurrence relation (2.1) is gotten from last two equations.

Let the skein operations be applied to a designated crossing of the clasp of the oriented diagram $\mathscr{K}_{n}$. The crossings of the clasp are right-handed and left-handed when $n$ is odd and even, respectively. In case of $n$ is odd, if the crossing is switched, the resulting diagram is an unknot by applying the second Reidemeister move and the first Reidemeister move $n$ times. Then, if the crossing is smoothed, the resulting diagram is $\mathscr{K}_{(2, n+1)}$ torus link with counter-directed strands taking into consideration $n+1$ is even. Hence, from the axiom (1.1) and (1.2), the relation in (2.2) is obtained as

$$
P_{n}=a z P_{\mathscr{K}_{(2, n+1)}}+a^{2} .
$$

In case of $n$ is even, the relation in (2.2) is obtained similarly.

Then, the recurrence relation (2.1) in Theorem 2.1 could be given with initial conditions as a fourth-order recurrence relation.

Definition 2.2. The HOMFLY polynomials $\left\{P_{n}\right\}_{n=0}^{\infty}$ for the oriented diagrams of twist knots $\mathscr{K}_{n}$ is defined by the recurrence relation

$$
P_{n}=\left(a^{2}+1\right) P_{n-2}-a^{2} P_{n-4}, \quad n \geq 4
$$

with initial conditions

$$
P_{0}=1, \quad P_{1}=a^{2} z^{2}-a^{4}+2 a^{2}, \quad P_{2}=a^{2}-z^{2}+a^{-2}-1, \quad P_{3}=a^{4} z^{2}+a^{2} z^{2}-a^{6}+a^{4}+a^{2} .
$$

Also, since $P_{K^{*}}(a, z)=P_{K}\left(a^{-1},-z\right)$, where $K^{*}$ is the mirror image of the diagram $K$, the following relation is obtained by using $P_{-n}$ instead of $P_{\mathscr{K}_{n}^{*}}(a, z)$

$$
P_{-n}=\left(a^{-2}+1\right) P_{-(n-2)}-a^{-2} P_{-(n-4)} .
$$

The characteristic equation of (2.1) is a bi-quadratic equation as

$$
\lambda^{4}-\left(a^{2}+1\right) \lambda^{2}+a^{2}=0
$$

and the roots of this equation are

$$
\lambda_{1}=a, \quad \lambda_{2}=-a, \quad \lambda_{3}=1, \quad \lambda_{4}=-1
$$


Proposition 2.3. The generating function of the sequence $\left\{P_{n}\right\}$ is

$$
g_{P}(\lambda)=\frac{-a^{2} \lambda^{3}+\left(a^{-2}-z^{2}-2\right) \lambda^{2}+\left(a^{2} z^{2}-a^{4}+2 a^{2}\right) \lambda+1}{a^{2} \lambda^{4}-\left(a^{2}+1\right) \lambda^{2}+1} .
$$

Proof. The generating function of $\left\{P_{n}\right\}$ has the following form:

$$
g_{P}(\lambda)=P_{0}+P_{1} \lambda+P_{2} \lambda^{2}+\ldots
$$

After the multiplications $\left(a^{2}+1\right) \lambda^{2} g_{P}(\lambda)$ and $-a^{2} \lambda^{4} g_{P}(\lambda)$, the following is provided by using (2.1)

$$
\begin{aligned}
\left(1-\left(a^{2}+1\right) \lambda^{2}+a^{2} \lambda^{4}\right) g_{P}(\lambda)= & P_{0}+P_{1} \lambda+\left(P_{2}-\left(a^{2}+1\right) P_{0}\right) \lambda^{2}+\left(P_{3}-\left(a^{2}+1\right) P_{1}\right) \lambda^{3} \\
& +\sum_{n=4}^{\infty}\left(P_{n}-\left(a^{2}+1\right) P_{n-2}+a^{2} P_{n-4}\right) \lambda^{n} \\
= & P_{0}+P_{1} \lambda+\left(P_{2}-\left(a^{2}+1\right) P_{0}\right) \lambda^{2}+\left(P_{3}-\left(a^{2}+1\right) P_{1}\right) \lambda^{3} .
\end{aligned}
$$

Hence, the equality (2.5) is obtained from the below by using the equalities in (2.3).

$$
g_{P}(\lambda)=\frac{P_{0}+P_{1} \lambda+\left(P_{2}-\left(a^{2}+1\right) P_{0}\right) \lambda^{2}+\left(P_{3}-\left(a^{2}+1\right) P_{1}\right) \lambda^{3}}{a^{2} \lambda^{4}-\left(a^{2}+1\right) \lambda^{2}+1} .
$$

Proposition 2.4. The general solution of the recurrence relation (2.1) is

$$
P_{n}=A a^{n}+B(-a)^{n}+C+D(-1)^{n}, \quad n \geq 0,
$$

where

$$
\begin{array}{cl}
A=-\frac{\left(a^{2}+a+1\right)\left(a^{4}-a^{2}\left(z^{2}+2\right)+1\right)}{2 a^{2}(a+1)}, & C=\frac{a^{4}-a^{2} z^{2}+1}{2 a^{2}}, \\
B=\frac{\left(a^{2}-a+1\right)\left(a^{4}-a^{2}\left(z^{2}+2\right)+1\right)}{2 a^{2}(a-1)}, & D=-\frac{\left(a^{2}+1\right)\left(a^{4}-a^{2}\left(z^{2}+2\right)+1\right)}{2 a^{2}\left(a^{2}-1\right)} .
\end{array}
$$

Proof. The closed form of the sequence $\left\{P_{n}\right\}$ is given by

$$
P_{n}=A \lambda_{1}^{n}+B \lambda_{2}^{n}+C \lambda_{3}^{n}+D \lambda_{4}^{n}, \quad n \geq 0 .
$$

Then, the following linear equation system is provided from (2.3) and (2.4) as

$$
\begin{aligned}
& P_{0}=A+B+C+D=1, \\
& P_{1}=A \lambda_{1}+B \lambda_{2}+C \lambda_{3}+D \lambda_{4}=a^{2} z^{2}-a^{4}+2 a^{2}, \\
& P_{2}=A \lambda_{1}^{2}+B \lambda_{2}^{2}+C \lambda_{3}^{2}+D \lambda_{4}^{2}=a^{2}-z^{2}+a^{-2}-1, \\
& P_{3}=A \lambda_{1}^{3}+B \lambda_{2}^{3}+C \lambda_{3}^{3}+D \lambda_{4}^{3}=a^{4} z^{2}+a^{2} z^{2}-a^{6}+a^{4}+a^{2} .
\end{aligned}
$$

The values $A, B, C$ and $D$ is obtained by solving this system. Note that considering $x^{n}-y^{n}=(x-y) \sum_{i=0}^{n-1} x^{k} y^{n-1-k}$, the factors $(a-1)$ are simplified.

Corollary 2.5. For $n \geq 2$, the explicit formula for the HOMFLY polynomial of twist knot $\mathscr{K}_{n}$ is given by

$$
P_{n}= \begin{cases}\frac{z^{2}}{a+1}\left(\sum_{i=0}^{n} a^{i+2}\right)-a^{n+3}+a^{n+1}+a^{2} & \text { if } n \text { is odd }, \\ -\frac{z^{2}}{a+1}\left(\sum_{i=0}^{n-1} a^{i}\right)+a^{n}-a^{n-2}+a^{-2} & \text { if } n \text { is even } .\end{cases}
$$

Proof. From Corollary 1 in [28], the explicit formula for the HOMFLY polynomial of $(2, n)$-torus link $\mathscr{K}_{(2, n)}$ with counterdirected strands taking into consideration that $n$ is even and the notations and diagrams mentioned in this paper is given by

$$
P_{\mathscr{K}_{(2, n)}}=\left(\frac{a^{n}-1}{a-a^{-1}}\right) z-a^{n}\left(a-a^{-1}\right) z^{-1} .
$$

Hence, the formulas in (2.6) are provided by using (2.2) and (2.7).

Remark 2.6. Since it is well known that the HOMFLY polynomial specializes to the Jones polynomial for a $=t$ and $z=t^{1 / 2}-t^{-1 / 2}$, the Alexander-Conway polynomial for $a=1$ and the Alexander polynomial for $a=1$ and $z=t^{1 / 2}-t^{-1 / 2}$, the recurrence relations for the mentioned knot polynomials of twist knot $\mathscr{K}_{n}$ could be easily obtained. 


\subsection{Kauffman polynomials $L$ and $F$ of twist knots}

Now, suppose that $\mathfrak{K}_{n}$ is an unoriented digram of twist knot drawn in Figure 2.1, $\mathfrak{K}_{(2, n)}$ is an unoriented digram of $(2, n)$-torus link drawn in Figure 2.2 and $L_{n}$ denotes the Kauffman polynomial $L$ of $\mathfrak{K}_{n}$ instead of $L_{\mathfrak{K}_{n}}(a, x)$ for simplicity.

Theorem 2.7. The Kauffman polynomial L of twist knot $\mathfrak{K}_{n}$ satisfies the following relations:

$$
L_{n}=x L_{n-1}+\left(a^{2}-1\right) L_{n-2}-a^{2} x L_{n-3}+a^{2} L_{n-4}, \quad n \geq 4
$$

and

$$
L_{n}=a^{-1} x L_{\mathfrak{K}_{(2, n)}}+x L_{\mathfrak{K}_{(2, n+1)}}-a^{n} .
$$

Proof. Let the axiom (1.3) be applied to a designated half twist of the unoriented diagram $\mathfrak{K}_{n}$. If the crossing is switched, the resulting diagram is $\mathfrak{K}_{n-2}$ twist knot by the second Reidemeister move. If the crossing is split according to the $K^{0}$, the resulting diagram is $\mathfrak{K}_{n-1}$ twist knot. Then, if the crossing is split according to the $K^{\infty}$, the resulting diagram is $(2,2)$-torus link, i.e. Hopf link, obtained by applying the first Reidemeister move $n-1$ times. Note that, if $n$ is even, all crossings of the (2,2)-torus link are left-handed and if $n$ is odd, all crossings of the (2,2)-torus link are right-handed. Hence, by using the axioms (1.4) and (1.5), the following equations are obtained as

$$
L_{n}=x L_{n-1}+a^{n-1} x L_{\mathfrak{K}_{(2,2)}}-L_{n-2}
$$

and

$$
L_{n-2}=x L_{n-3}+a^{n-3} x L_{\mathfrak{K}_{(2,2)}}-L_{n-4}
$$

Thus, the recurrence relation (2.8) is gotten from last two equations.

Let the axiom (1.3) be applied to a designated crossing of the clasp of the unoriented diagram $\mathfrak{K}_{n}$. If the crossing is switched, the resulting diagram is an unknot obtained by applying the second Reidemeister move and the first Reidemeister move $n$ times. If the crossing is split according to the $K^{0}$, the resulting diagram is torus link $\mathfrak{K}_{(2, n+1)}$. Then, if the crossing is split according to the $K^{\infty}$, the resulting diagram is the image of torus link $\mathfrak{K}_{(2, n)}$ by applying the first Reidemeister move. Thus, the relation (2.9) is obtained by using the axioms (1.4), (1.5) and (1.6).

Then, the recurrence relation (2.8) in Theorem 2.7 could be given with initial conditions as a fourth-order recurrence relation.

Definition 2.8. The Kauffman polynomials $\left\{L_{n}\right\}_{n=0}^{\infty}$ for the unoriented diagrams of twist knots $\mathfrak{K}_{n}$ is defined by the recurrence relation

$$
L_{n}=x L_{n-1}+\left(a^{2}-1\right) L_{n-2}-a^{2} x L_{n-3}+a^{2} L_{n-4}, \quad n \geq 4
$$

with initial conditions

$$
\begin{gathered}
L_{0}=a^{-2}, \quad L_{1}=\left(a+a^{-1}\right) x^{2}+\left(a^{-2}+1\right) x-2 a-a^{-1}, \\
L_{2}=\left(a+a^{-1}\right) x^{3}+\left(a^{2}+a^{-2}+2\right) x^{2}-\left(a+a^{-1}\right) x-a^{2}-a^{-2}-1, \\
L_{3}=\left(a+a^{-1}\right) x^{4}+\left(a^{2}+a^{-2}+2\right) x^{3}+\left(a^{3}-a-2 a^{-1}\right) x^{2}-\left(2 a^{-2}+2\right) x-a^{3}+a+a^{-1} .
\end{gathered}
$$

Since $L_{K^{*}}(a, x)=L_{K}\left(a^{-1}, x\right)$, where $K^{*}$ is the mirror image of the diagram $K$, the following relation is obtained by using $L_{-n}$ instead of $L_{\mathscr{K}_{n}^{*}}(a, x)$

$$
L_{-n}=x L_{-(n-1)}+\left(a^{-2}-1\right) L_{-(n-2)}-a^{-2} x L_{-(n-3)}+a^{-2} L_{-(n-4)} .
$$

The characteristic equation of (2.8) is a quadratic equation as

$$
\lambda^{4}-x \lambda^{3}-\left(a^{2}-1\right) \lambda^{2}+a^{2} x \lambda-a^{2}=0
$$

and the roots of this equation are

$$
\lambda_{1}=a, \quad \lambda_{2}=-a, \quad \lambda_{3}=\frac{1}{2}\left(x+\sqrt{x^{2}-4}\right), \quad \lambda_{4}=\frac{1}{2}\left(x-\sqrt{x^{2}-4}\right) .
$$

Proposition 2.9. The generating function of the sequence $\left\{L_{n}\right\}$ is

$$
g_{L}(\lambda)=\frac{a^{3} \lambda^{3}+\left(\left(a^{2}+1\right) x^{2}+a x-a^{2}-2\right) \lambda^{2}+\left(\left(a+a^{-1}\right) x^{2}+x-2 a-a^{-1}\right) \lambda+a^{-2}}{-a^{2} \lambda^{4}+a^{2} x \lambda^{3}-\left(a^{2}-1\right) \lambda^{2}-x \lambda+1} .
$$


Proof. The generating function of $\left\{L_{n}\right\}$ has the following form:

$$
g_{L}(\lambda)=L_{0}+L_{1} \lambda+L_{2} \lambda^{2}+\ldots
$$

After the multiplications $x \lambda g_{L}(\lambda),\left(a^{2}-1\right) \lambda^{2} g_{L}(\lambda),-a^{2} x \lambda^{3} g_{L}(\lambda)$ and $a^{2} \lambda^{4} g_{L}(\lambda)$, the following is provided by using (2.8)

$$
\begin{aligned}
\left(1-x \lambda-\left(a^{2}-1\right) \lambda^{2}+a^{2} x \lambda^{3}-a^{2} \lambda^{4}\right) g_{L}(\lambda)= & L_{0}+\left(L_{1}-x L_{0}\right) \lambda+\left(L_{2}-x L_{1}-\left(a^{2}-1\right) L_{0}\right) \lambda^{2}+\left(L_{3}-x L_{2}-\left(a^{2}-1\right) L_{1}+a^{2} x L_{0}\right) \lambda^{3} \\
& +\sum_{n=4}^{\infty}\left(L_{n}-x L_{n-1}-\left(a^{2}-1\right) L_{n-2}+a^{2} x L_{n-3}-a^{2} L_{n-4}\right) \lambda^{n} \\
= & L_{0}+\left(L_{1}-x L_{0}\right) \lambda+\left(L_{2}-x L_{1}-\left(a^{2}-1\right) L_{0}\right) \lambda^{2}+\left(L_{3}-x L_{2}-\left(a^{2}-1\right) L_{1}+a^{2} x L_{0}\right) \lambda^{3}
\end{aligned}
$$

Hence, the equality (2.5) is obtained from the below by using the equalities in (2.3).

$$
g_{L}(\lambda)=\frac{L_{0}+\left(L_{1}-x L_{0}\right) \lambda+\left(L_{2}-x L_{1}-\left(a^{2}-1\right) L_{0}\right) \lambda^{2}+\left(L_{3}-x L_{2}-\left(a^{2}-1\right) L_{1}+a^{2} x L_{0}\right) \lambda^{3}}{-a^{2} \lambda^{4}+a^{2} x \lambda^{3}-\left(a^{2}-1\right) \lambda^{2}-x \lambda+1} .
$$

Proposition 2.10. The general solution of the recurrence relation (2.8) is

$$
L_{n}=A a^{n}+B(-a)^{n}+C\left(\frac{1}{2}\left(x+\sqrt{x^{2}-4}\right)\right)^{n}+D\left(\frac{1}{2}\left(x-\sqrt{x^{2}-4}\right)\right)^{n}, \quad n \geq 0,
$$

where

$$
\begin{aligned}
& A=\frac{a^{2}\left(x^{2}-1\right)+a x+x^{2}-1}{a^{2}-a x+1}, \quad B=0 \\
& C=\frac{\left(a^{2}+1\right)\left(2 a^{3}+a^{2}\left(x^{3}+x^{2} \sqrt{x^{2}-4}-\sqrt{x^{2}-4}-3 x\right)+a\left(-x^{2}+x \sqrt{x^{2}-4}+2\right)-\sqrt{x^{2}-4}-x\right)}{2 a^{2} \sqrt{x^{2}-4}\left(-a^{2}+a x-1\right)}, \\
& D=\frac{\left(a^{2}+1\right)\left(-2 a^{3}+a^{2}\left(-x^{3}+x^{2} \sqrt{x^{2}-4}-\sqrt{x^{2}-4}+3 x\right)+a\left(x^{2}+x \sqrt{x^{2}-4}-2\right)-\sqrt{x^{2}-4}+x\right)}{2 a^{2} \sqrt{x^{2}-4}\left(-a^{2}+a x-1\right)} .
\end{aligned}
$$

Proof. The closed form of the sequence $\left\{L_{n}\right\}$ is given by

$$
L_{n}=A \lambda_{1}^{n}+B \lambda_{2}^{n}+C \lambda_{3}^{n}+D \lambda_{4}^{n}, \quad n \geq 0 .
$$

Then, the following linear equation system is provided from (2.10) and (2.11) as

$$
\begin{aligned}
& L_{0}=A+B+C+D=a^{-2}, \\
& L_{1}=A \lambda_{1}+B \lambda_{2}+C \lambda_{3}+D \lambda_{4}=\left(a+a^{-1}\right) x^{2}+\left(a^{-2}+1\right) x-2 a-a^{-1}, \\
& L_{2}=A \lambda_{1}^{2}+B \lambda_{2}^{2}+C \lambda_{3}^{2}+D \lambda_{4}^{2}=\left(a+a^{-1}\right) x^{3}+\left(a^{2}+a^{-2}+2\right) x^{2}-\left(a+a^{-1}\right) x-a^{2}-a^{-2}-1, \\
& L_{3}=A \lambda_{1}^{3}+B \lambda_{2}^{3}+C \lambda_{3}^{3}+D \lambda_{4}^{3}=\left(a+a^{-1}\right) x^{4}+\left(a^{2}+a^{-2}+2\right) x^{3}+\left(a^{3}-a-2 a^{-1}\right) x^{2}-\left(2 a^{-2}+2\right) x-a^{3}+a+a^{-1} .
\end{aligned}
$$

The values $A, B, C$ and $D$ is obtained by solving this system.

Suppose that $F_{n}$ denotes the Kauffman polynomial $F$ of $\mathfrak{K}_{n}$ instead of $F_{\mathfrak{K}_{n}}(a, x)$ for simplicity.

Corollary 2.11. The Kauffman polynomials $\left\{F_{n}\right\}_{n=0}^{\infty}$ for the unoriented diagrams of twist knots $\mathfrak{K}_{n}$ is defined by the recurrence relation

$$
F_{n}=\left\{\begin{array}{ll}
a^{-5} x F_{n-1}+\left(1-a^{-2}\right) F_{n-2}-a^{-5} x F_{n-3}+a^{-2} F_{n-4} & \text { if } n \text { is odd, } \\
a^{3} x F_{n-1}+\left(1-a^{-2}\right) F_{n-2}-a^{3} x F_{n-3}+a^{-2} F_{n-4} & \text { if } n \text { is even, }
\end{array} \quad n \geq 4\right.
$$

with initial conditions

$$
\begin{gathered}
F_{0}=1, \quad F_{1}=\left(a^{-2}+a^{-4}\right) x^{2}+\left(a^{-3}+a^{-5}\right) x-2 a^{-2}-a^{-4}, \\
F_{2}=\left(a+a^{-1}\right) x^{3}+\left(a^{2}+a^{-2}+2\right) x^{2}-\left(a+a^{-1}\right) x-a^{2}-a^{-2}-1, \\
F_{3}=\left(a^{-4}+a^{-6}\right) x^{4}+\left(a^{-3}+a^{-7}+2 a^{-5}\right) x^{3}+\left(a^{-2}+-a^{-4}-2 a^{-6}\right) x^{2}-\left(2 a^{-7}+2 a^{-5}\right) x-a^{-2}+a^{-4}+a^{-6} .
\end{gathered}
$$

Also, the following relation is satisfied for $F_{n}$.

$$
F_{n}= \begin{cases}a^{-3} x F_{\mathfrak{K}_{(2, n)}}+a^{-1} x F_{\mathfrak{K}_{(2, n+1)}}-a^{-2} & \text { if } n \text { is odd }, \\ a x F_{\mathfrak{K}_{(2, n)}}+a^{3} x F_{\mathfrak{K}_{(2, n+1)}}-a^{2} & \text { if } n \text { is even } .\end{cases}
$$


Proof. It is proven by considering $w\left(\mathfrak{K}_{n}\right)=n+2$ and $w\left(\mathfrak{K}_{n}\right)=n-2$ if $n$ is odd and even, respectively, and by using (1.7) in Definition 2.8 and the relation (2.9).

In addition, since $F_{K^{*}}(a, x)=F_{K}\left(a^{-1}, x\right)$, where $K^{*}$ is the mirror image of the diagram $K$, the following relations are obtained by using $F_{-n}$ instead of $F_{\mathscr{K}_{n}^{*}}(a, x)$

$$
F_{-n}= \begin{cases}a^{5} x F_{-(n-1)}+\left(1-a^{2}\right) F_{-(n-2)}-a^{5} x F_{-(n-3)}+a^{2} F_{-(n-4)} & \text { if } n \text { is odd } \\ a^{-3} x F_{-(n-1)}+\left(1-a^{2}\right) F_{-(n-2)}-a^{-3} x F_{-(n-3)}+a^{2} F_{-(n-4)} & \text { if } n \text { is even }\end{cases}
$$

By using same notation, it could be provided a relation from (2.14) for the mirror image of $\mathscr{K}_{n}^{*}$.

Corollary 2.12. The general solution of the recurrence relation (2.13) is

$$
F_{n}= \begin{cases}a^{-n-2}\left(A a^{n}+B(-a)^{n}+C\left(\frac{1}{2}\left(x+\sqrt{x^{2}-4}\right)\right)^{n}+D\left(\frac{1}{2}\left(x-\sqrt{x^{2}-4}\right)\right)^{n}\right) & \text { if } n \text { is odd }, \\ a^{-n+2}\left(A a^{n}+B(-a)^{n}+C\left(\frac{1}{2}\left(x+\sqrt{x^{2}-4}\right)\right)^{n}+D\left(\frac{1}{2}\left(x-\sqrt{x^{2}-4}\right)\right)^{n}\right) & \text { if } n \text { is even, }\end{cases}
$$

where

$$
\begin{aligned}
& A=\frac{a^{2}\left(x^{2}-1\right)+a x+x^{2}-1}{a^{2}-a x+1}, \quad B=0 \\
& C=\frac{\left(a^{2}+1\right)\left(2 a^{3}+a^{2}\left(x^{3}+x^{2} \sqrt{x^{2}-4}-\sqrt{x^{2}-4}-3 x\right)+a\left(-x^{2}+x \sqrt{x^{2}-4}+2\right)-\sqrt{x^{2}-4}-x\right)}{2 a^{2} \sqrt{x^{2}-4}\left(-a^{2}+a x-1\right)}, \\
& D=\frac{\left(a^{2}+1\right)\left(-2 a^{3}+a^{2}\left(-x^{3}+x^{2} \sqrt{x^{2}-4}-\sqrt{x^{2}-4}+3 x\right)+a\left(x^{2}+x \sqrt{x^{2}-4}-2\right)-\sqrt{x^{2}-4}+x\right)}{2 a^{2} \sqrt{x^{2}-4}\left(-a^{2}+a x-1\right)} .
\end{aligned}
$$

Proof. The proof follows directly from (1.7) and (2.12) by considering $w\left(\mathfrak{K}_{n}\right)=n+2$ and $w\left(\mathfrak{K}_{n}\right)=n-2$ if $n$ is odd and even, respectively.

Remark 2.13. Since it is well known that the Kauffman polynomial $F$ specializes to the Jones polynomial for $a=-t^{3 / 2}$ and $x=t^{-1 / 4}+t^{1 / 4}$ and the BLM/Ho polynomial for $a=1$, the recurrence relations for the mentioned knot polynomials of twist knot $\mathfrak{K}_{n}$ could be easily obtained.

Corollary 2.14. For $n \geq 1$, the explicit form of of $\left\{L_{n}\right\}$ and $\left\{F_{n}\right\}$ are

$$
L_{n}=a^{-1} x R_{n+2}+\left(a x^{2}+a^{-2} x-a-a^{-1}\right) R_{n+1}+\left(x^{2}-a x+a^{2}-a^{-2}\right) R_{n}+\left(-x+a+a^{-1}\right) R_{n-1}-a^{n}
$$

and

$F_{n}=\left\{\begin{array}{l}a^{-1} x S_{n+2}+\left(a^{2} x^{2}+a^{-3} x-a^{2}-1\right) S_{n+1}+\left(x^{2}-a^{3} x+a^{4}+a^{2}-a^{-2}-1\right) S_{n}+\left(-a x+a^{2}+1\right) S_{n-1}-a^{-2} \text { if } n \text { is odd }, \\ a^{3} x S_{n+2}+\left(a^{6} x^{2}+a x-a^{6}-a^{4}\right) S_{n+1}+\left(a^{4} x^{2}-a^{7} x+a^{8}+a^{6}-a^{4}-a^{2}\right) S_{n}+\left(-a^{5} x+a^{6}+a^{4}\right) S_{n-1}-a^{2} \text { if } n \text { is even }\end{array}\right.$

where $\left\{R_{n}\right\}$ and $\left\{S_{n}\right\}$ are special cases of the following sequence $\left\{G_{n}\right\}$ with initial conditions $G_{0}=G_{1}=0, G_{2}=1$ for $r=a+x, s=-(1+a x), t=a$ and $r=a^{2}+a x, s=-\left(a^{2}+a^{3} x\right), t=a^{4}$, respectively.

$$
\left.G_{n}=\sum_{i=0}^{\left\lfloor\frac{n-2}{2}\right\rfloor\left\lfloor\frac{n-2}{3}\right\rfloor} \sum_{j=0}^{n-2-i-2 j} \begin{array}{c}
n+j \\
i+j
\end{array}\right)\left(\begin{array}{c}
i+j \\
j
\end{array}\right) r^{n-2-2 i-3 j} s^{i}(-1)^{i} t^{j}
$$

Proof. From Theorem 2.3 and 2.4 in [31], the explicit forms of the Kauffman polynomial $L$ and $F$ sequences of $\mathfrak{K}_{(2, n)}$ are given by

$$
L_{\mathfrak{K}_{(2, n)}}=\left(a^{-1}\right) R_{n+1}+\left(a x-\left(a+a^{-1}\right) x^{-1}\right) R_{n}+\left(\left(1+a^{2}\right) x^{-1}-a\right) R_{n-1}, \quad n \geq 1
$$

and

$$
F_{\mathfrak{K}_{(2, n)}}=S_{n+1}+\left(a^{3} x-\left(a^{3}+a\right) x^{-1}\right) S_{n}+\left(\left(a^{5}+a^{3}\right) x^{-1}-a^{4}\right) S_{n-1}, \quad n \geq 1 .
$$

Hence, the explicit form (2.15) is obtained by using (2.9) and (2.17). Then, the explicit form (2.16) is obtained by considering $w\left(\mathfrak{K}_{n}\right)=n+2$ and $w\left(\mathfrak{K}_{n}\right)=n-2$ if $n$ is odd and even, respectively, and by using (2.14) and (2.18). 


\section{References}

[1] J. W. Alexander, Topological invariants of knots and links, Trans. Amer. Math. Soc., 30 (1928), $275-308$.

[2] J. H. Conway, An enumeration of knots and kinks, and some of their algebraic properties, in Computational Problems in Abstract Algebra, (Pergamon, Oxford, 1970), 329-358.

[3] S. Friedl, S. Vidussi, A Survey of Twisted Alexander Polynomials, M. Banagl, D. Vogel (eds), The Mathematics of Knots. Contributions in Mathematical and Computational Sciences, Springer, Berlin, 2011, pp. 45-94.

[4] İ. Altıntaş, K. Taşköprü, A generalization of the Alexander polynomial, Int. J. Math. Comb., 4 (2016), 21-28.

[5] I. Altıntaş, K. Taşköprü, A generalization of the Alexander polynomial as an application of the delta derivative, Turk. J. Math., $42(2)$ (2018), 515-527.

[6] V. F. R. Jones, A polynomial invariant for knots via von Neumann algebras, Bull. Amer. Math. Soc., 12 (1985), $103-111$.

[7] L. H. Kauffman, State models and the Jones polynomial, Topology, 26 (1987), 395-407.

[8] I. Altıntaş, An oriented state model for the Jones polynomial and its applications alternating links, Appl. Math. Comput., 194(1) (2007), 168-178.

[9] P. M. Melvin, H. R. Morton, The coloured Jones function, Commun. Math. Phys, 169 (1995), 501-520.

[10] İ. Altıntaş, Computer algebra and colored Jones polynomials, Appl. Math. Comput., 182(1) (2006), 804-808.

[11] P. Freyd, D. Yetter, J. Hoste, W. B. R. Lickorish, K. Millett, A. Ocneau, A new polynomial invariant of knots and links, Bull. Amer. Math. Soc., 12 (1985), 239-246.

[12] J. H. Przytycki, P. Traczyk, Conway algebras and skein equivalence of links, Proc. Amer. Math. Soc., 100(4) (1987), 744-748.

[13] H. Doll, J. Hoste, A tabulation of oriented links, Math. Comp., 57(196) (1991), 747-761.

[14] R. D. Brandt, W. B. R. Lickorish, K. C. Millett, A polynomial invariant for unoriented knots and links, Invent. Math., 84 (1986), $563-573$.

[15] C. F. Ho, A new polynomial for knots and links-preliminary report, Abstracts Amer. Math. Soc., 6 (1985), 300.

[16] L. H. Kauffman, On knots, vol. 115 of Annals of Mathematics Study, Princeton University Press, New Jersey, 1987.

[17] L. H. Kauffman, An invariant of regular isotopy, Trans. Amer. Math. Soc., 318 (1990), 417-471.

[18] Y. Allin, M. E. Bozhüyük, The group of twist knots, Math. Comput. Appl., 1(2) (1996), 7-15.

[19] J. Hoste, P. D. Shanahan, Trace fields of twist knots, J. Knot Theory Ramifications, 10(4) (2001), 625-639.

[20] J. Hoste, P. D. Shanahan, A formula for the A-polynomial of twist knots, J. Knot Theory Ramifications, 13(2) (2004), 193-209.

[21] J. Dubois, V. Huynh, Y. Yamaguchi, Non-abelian Reidemeister torsion for twist knots, J. Knot Theory Ramifications, 18(3) (2009), 303-341.

[22] S. Garoufalidis, X. Sun, The non-commutative A-polynomial twist knots, J. Knot Theory Ramifications, 19(12) (2010), 1571-1595.

[23] D. V. Mathews, An explicit formula for the A-polynomial of twist knots, J. Knot Theory Ramifications, 23(9) (2014), 1450044, 5 pages.

[24] A. T. Tran, Reidemeister torsion and Dehn surgery on twist knots, Tokyo J. Math., 39(2) (2016), 517-526.

[25] A. Sahin, B. Sahin, Jones polynomial for graphs of twist knots, Appl. Appl. Math., 14(2) (2019), 1269-1278.

[26] B. Berceanu, A. R. Nizami, A recurrence relation for the Jones polynomial, J. Korean Math. Soc., 51 (2014), 443-462.

[27] P.-V. Koseleff, D. Pecker, On Alexander-Conway polynomials of two-bridge links, J. Symbolic Comput., 68 (2015), $215-229$.

[28] S. Duzhin, M. Shkolnikov, A formula for the HOMFLY polynomial of rational links, Arnold Math. J., 1 (2015), $345-359$.

[29] K. Taşköprü, İ. Altıntaş, HOMFLY polynomials of torus links as generalized Fibonacci polynomials, Electron. J. Combin., 22 (2015), 4.8.

[30] İ. Altıntaş, K. Taşköprü, M. Beyaztaş, Bracket polynomials of torus links as Fibonacci polynomials, Int. J. Adv. Appl. Math. and Mech., 5(3) (2018), 35-43.

[31] İ. Altıntaş, K. Taşköprü, Unoriented knot polynomials of torus links as Fibonacci-type polynomials, Asian-Eur. J. Math., 12(1) (2019), 1950053, 17 pages. 